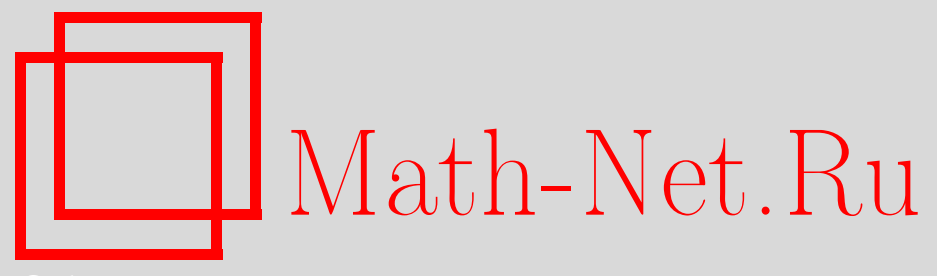

С. А. Степанов, М. Х. Шалалфех, Коды на расслоенных произведениях кривых Артина-Шрейера, Дискрет. матем., 2001, том 13, выпуск 2, 3-13

DOI: https://doi.org/10.4213/dm287

Использование Общероссийского математического портала Math-Net.Ru подразумевает, что вы прочитали и согласны с пользовательским соглашением http://www . mathnet.ru/rus/agreement

Параметры загрузки:

IP : 54.196 .121 .252

26 апреля 2023 г., 13:46:45 
УДК 519.4

\title{
Коды на расслоенных произведениях кривых Артина-Шрейера
}

\author{
(C) 2001 г. С. А. Степанов, М. Х. Шалалфех
}

В статье с использованием расслоенного произведения кривых АртинаШрейера построено новое семейство гладких проективных кривых над конечным полем $F_{q}$ с большим числом $F_{q}$-рациональных точек. Показано, что для любой кривой $X$ из этого семейства отношение $g(X) / N_{q}(X)$, где $g(X)$ - род и $N_{q}(X)$ - число $F_{q}$-рациональных точек, достаточно мало, чтобы получить геометрические коды Гоппы с хорошими параметрами. В статье обобщаются результаты С. А. Степанова и $\Phi$. Озбудака о построении кодов большой длины.

\section{1. Введение}

Пусть $p$ - простое число и $F_{q}$ - конечное поле с $q=p^{\nu}$ элементами, $\nu$ - натуральное число. Линейный $[n, k, d]_{q}$-код $C$ - это подпространство пространства $F_{q}^{n}$, где $n-$ длина кода $C, k=\operatorname{dim}_{F_{q}} C-$ размерность $C$ и $d-$ кодовое расстояние, минимальное расстояние Хемминга между ненулевыми элементами $C$. Каждый линейный $[n, k, d]$-код $C$ определяет пару относительных параметров $(\delta, R)$, где $\delta=d / n$ - относительное минимальное расстояние и $R=k / n$ - скорость передачи кода $C$.

Используя конструкцию Гоппы линейных кодов, связанную с гладкими проективными кривыми над конечными полями, можно доказать существование линейньх кодов большой длины. Хотя геометрические коды Гоппы, связанные с максимальными кривыми, обладают хорошими относительными параметрами, длина этих кодов ограничена величиной $1+q^{3}$. Эта оценка является прямым следствием оценки ХассеВейля и того факта, что род максимальной кривой $X$ удовлетворяет неравенству $g(X) \leqslant q(q-1) / 2$ (см. [10]).

В статье [7] С. А. Степанов построил геометрические коды Гоппы большой длины с использованием расслоенного произведения гиперэллиптических кривых над конечным полем $F_{q}$, для которого $q=p^{\nu}$ и $\nu$ - четное число. В [8] С. А. Степановым и $\Phi$. Озбудаком этот результат обобщен на случай, когда $\nu$ нечетно. В [4] Ф. Озбудак с использованием расслоенного произведения покрытий Куммера построил коды, длина которых больше длин кодов, построенных в [7] и [8]. В настоящей статье с использованием расслоенных произведений кривых Артина-Шрейера строятся геометрические коды Гоппы, длина которых значительно длиннее кодов из статей [6], [7], [8] и [4]. 
Далее $F_{q}[x]$ обозначает пространство рациональньх функций проективной прямой $P^{1}$. Элемент $f(x) \in F_{q}[x]$ называется вырожденным если существует такой элемент $g(x) \in F_{q}[x]$, что $f=g^{p}-g+a$, где $a \in F_{q}$. В противном случае элемент $f$ называется невырожденным.

Если $C$ - код над $F_{q}$ и $\operatorname{Tr}_{\nu}: F_{q} \rightarrow F_{p}$ обозначает функцию след из $F_{q}$ в $F_{p}$, то

$$
\operatorname{Tr}(C)=\left\{\operatorname{Tr}_{\nu}(c) \mid c \in C\right\}
$$

- код над $F_{p}$, называемый след-кодом кода $C$.

Соотношение $\operatorname{Tr}_{\nu}(\alpha)=0$, выполняемое тогда и только тогда, когда существует такое $\beta \in F_{q}$, что $\beta^{p}-\beta=\alpha$, связывает след-коды с кривыми Артина-Шрейера $X_{f}$ с определяющим уравнением

$$
y^{p}-y=f(x) \in F_{q}(x) .
$$

Кривая (1) абсолютно неприводима тогда и только тогда, когда кривая $f(x)$ невырождена (см. [3]). Известно, что если $f(x) \in F_{q}[x], \operatorname{deg} f(x)=m$ и $(m, p)=1$, то $f(x)$ невырождена (см. [5]).

Обозначим $N_{q}\left(X_{f}\right)$ число $F_{q}$-рациональных точек гладкой проективной модели кривой (1), это число равно

$$
N_{q}\left(X_{f}\right)=p N+1
$$

где $N=\left|\left\{x \in F_{q}: \operatorname{Tr}_{\nu} f(x)=0\right\}\right|$.

Пусть $A-F_{q}$-линейное подпространство пространства $F_{q}[x], A$ называется невырожденным, если каждая функция $f(x) \in A$ невырождена. Если рассматривать $A$ как векторное пространство над $F_{p}$, то ясно, что существует базис $\left\{f_{1}(x), \ldots, f_{s}(x)\right\}$ пространства $A$ над $F_{p}$ и

$$
A=F_{p} f_{1}(x)+\ldots+F_{p} f_{s}(x) .
$$

Такой базис будем называть каноническим, если полиномы любого подмножества этого базиса, состоящего из полиномов одинаковых степеней, имеют $F_{p}$-линейно независимые коэффициенты при старшем члене. В таком случае система уравнений над $\bar{F}_{q}$

$$
\begin{gathered}
y_{1}^{p}-y_{1}=f_{1}(x) \\
y_{2}^{p}-y_{2}=f_{2}(x) \\
\ldots \\
y_{s}^{p}-y_{s}=f_{s}(x)
\end{gathered}
$$

определяет неприводимую кривую в $(s+1)$-мерном проективном пространстве $P^{s+1}$ (см. [3]).

В [9] рассмвтривались расслоенные произведения

$$
y_{i}^{p}-y_{i}=a_{i} x^{\sqrt{q}+1},
$$

где $a_{i} \in F_{q}^{*}$ Удовлетворяют равенству $a_{i}^{\sqrt{q}}+a_{i}=0$, если $q=p^{\nu}$ и $\nu$ четно, и

$$
y_{i}^{p}-y_{i}=a_{i} x^{p^{(\nu+1) / 2}+1}-a_{i}^{(\nu-1) / 2} x^{p^{(\nu-1) / 2}+1},
$$


где $a_{i} \in F_{q}^{*}$, если $q=p^{\nu}$ и $\nu$ нечетно.

В результате в [9] были получены гладкие проективные кривые $X_{r}$ такие, что

$$
g\left(X_{r}\right)= \begin{cases}\left(p^{r}-1\right) \sqrt{q} / 2, & 1 \leqslant r \leqslant \nu / 2, \nu \text { четно, } \\ \left(p^{r}-1\right) \sqrt{p q} / 2, & 1 \leqslant r \leqslant \nu, \nu \text { нечетно, }\end{cases}
$$

и число $F_{q}$-рациональных точек кривой $X_{r}$ равно

$$
N_{q}\left(X_{r}\right)= \begin{cases}p^{r} q+1, & 1 \leqslant r \leqslant \nu / 2, \nu \text { четно, } \\ p^{r} q+1, & 1 \leqslant r \leqslant \nu, \nu \text { нечетно. }\end{cases}
$$

Замечание 1. Для четного $\nu$ кривая $X_{r}$ является максимальной кривой при любом $r, 1 \leqslant r \leqslant \nu / 2$.

В этой статье мы строим семейство невырожденных полиномов $f_{i}(x) \in F_{q}[x]$, образующих канонический базис линейного подпространства пространства $F_{q}[x]$, и к этому семейству полиномов применяем расслоенное произведение (2) для получения гладких проективных кривых над конечным полем, имеющих большое число рациональных точек и малое отношение $g(X) / N_{q}(X)$. Получены следующие результаты.

Теорема 1. Пусть $q=p^{\nu} u F_{q^{2}}$ - конечное поле $c q^{2}$ элементами. Тогда для любого челого $s, 1 \leqslant s<q,(s, p)=1$, существует гладкая проективная кривая $X_{s}$, для которой род $g\left(X_{s}\right)$ и иисло $F_{q^{2}}$-рачионалвнвх точех $N_{q}\left(X_{s}\right)$ удовлетворяют соотношениям

$$
g\left(X_{s}\right) \leqslant \frac{1}{2}\left(q^{N_{1}(s)}-1\right)(s(q+1)-1), \quad N_{q}\left(X_{s}\right)=q^{N_{1}(s)+2}+1
$$

где $N_{1}(s)=s-[s / p]+2\left[\log _{p}((s(q+1)-1) / q)\right]$.

Теорема 2. Пусть $q=p^{2 \nu+1}$ u $F_{q}$ - конечное поле с $q$ элементами. Тогда для любого челого $s, 1 \leqslant s<p^{\nu-1},(s, p)=1$, существует гладкал проективная кривал $X_{s}$, для которой род $g\left(X_{s}\right)$ и число $F_{q}$-рачионалънъх точек удовлетворяют соотношениям

$$
g\left(X_{s}\right)<\frac{1}{2}\left(p^{N_{2}(s)}-1\right)\left(s\left(p^{\nu+1}+1\right)-1\right), \quad N_{q}\left(X_{s}\right)=q p^{N_{2}(s)}+1,
$$

где $N_{2}(s)=(2 \nu+1)(s-[s / p])$.

Применяя конструкцию Гоппы к семейству кривых, описанных в этих теоремах, получаем следующие результаты.

Следствие 1. Пусть $F_{q^{2}}, s, N_{1}(s)$ - такие, как в теореме 1. Тогда для любого

$$
l>\frac{1}{2}\left(q^{N_{1}(s)}-1\right)(s(q+1)-1)
$$

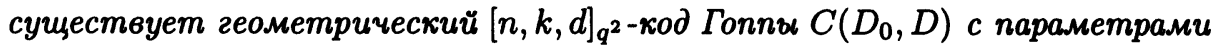

$$
\begin{aligned}
& l<n \leqslant q^{N_{1}(s)+2}, \\
& k \geqslant l-\frac{1}{2}\left(q^{N_{1}(s)}-1\right)(s(q+1)-1)+1, \\
& d \geqslant n-l
\end{aligned}
$$


Следствие 2. Пусть $F_{q}, s, N_{2}(s)$ - такие, ках в теореме 2. Тогда для азобого

$$
l>\frac{1}{2}\left(p^{N_{2}(s)}-1\right)\left(s\left(p^{\nu+1}+1\right)-1\right)
$$

существует геометрический $[n, k, d]_{q}-$ код Гоппы $C\left(D_{0}, D\right)$ с параметрами

$$
\begin{aligned}
& l<n \leqslant q p^{N_{2}(s)} \\
& k \geqslant l-\frac{1}{2}\left(p^{N_{2}(s)}-1\right)\left(s\left(p^{\nu+1}+1\right)-1\right)+1, \\
& d \geqslant n-l
\end{aligned}
$$

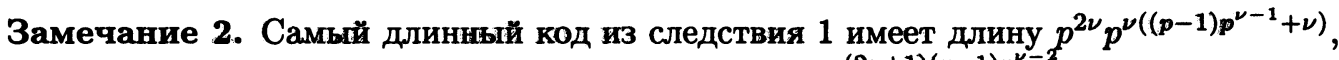
а длина самого длинғого кода шз теоремы 2 равна $p^{(2 \nu+1)(p-1) p^{\nu-2}}$, эти коды значительно длиннее, чем коды из статьи [4], где улучшены результаты статей [7] и [8]. Самый длинный код, описанғый в [4] имеет длину $(p-1)^{p^{\nu}} p^{\nu}$.

Замечание 3. Относительные параметры $R=k / n$ и $\delta=d / n$ кодов из следствий 1 и 2 удовлетворяют, соответственно, неравенствам

$$
\begin{aligned}
& R \geqslant 1-\delta-\frac{\left(q^{N_{1}(s)}-1\right)(s(q+1)-1)-1}{q^{N_{1}(s)+2}+1}, \\
& R \geqslant 1-\delta-\frac{\left(p^{N_{2}(s)}-1\right)\left(s\left(p^{\nu+1}+1\right)-1\right)-1}{q p^{N_{2}(s)}+1} .
\end{aligned}
$$

Доказательство основњых результатов этой статьи проводится следующий образом. В параграфе 2 приводатся явное выражение для рода кривой (2). В параграфе 3 строится канонический базис невырожденного подпространства пространства $F_{q}[x]$ и находится число $F_{q}$-рациянальных точек кривой, определенной системой (2) для этого канонического базиса. В последнем параграфе доказываются теоремы 1 и 2 , а также их следствия.

\section{2. Вычисление рода кривой}

Пусть $F_{q}$ - конечное поле характеристики $p$ с $q=p^{\nu}$ элементами. Пусть $f(x) \in F_{q}[x]$ - полином степени $m,(m, p)=1$. Тогда кривая $X$, определенная над $\bar{F}_{q}$ уравнением

$$
y^{p}-y=f(x)
$$

неприводима. Следующая лемма хорошо известна; мы приводим ее доказательство, поскольку его часть используется в доказательстве теоремы 3.

Лемма 1. Род кривой $X$ равен

$$
g(X)=\frac{1}{2}(p-1)(m-1) .
$$

Доказательство. Кривая $X$ определяет покрытие $\phi: X \rightarrow P^{1}$ степени $p$ проективной прямой $P^{1}$. Единственной точкой ветвления покрытия $\phi$ является точка на бесконечности. Пусть $p_{\infty}=[0: 1]-$ точка на бесконечности и $x$ - координата в $A^{1}$. Если взять $t=1 / x$ в качестве локального параметра в $p_{\infty}$, то обратным образом $p_{\infty}$ будет точка $x_{\infty}$ на кривой $X$, которая соответствует дискретной оценке $v_{x_{\infty}} \mathrm{c}$ 
$v_{x_{\infty}}(x)=-p$ и $v_{x_{\infty}}(y)=-m$. Локальный параметр $x_{\infty}$ есть $s=1 /\left(x^{a} y^{b}\right)$, где $a p+b m=1$. Так как $v_{x_{\infty}}\left(1 /\left(x^{a} y^{b}\right)\right)=1$,

$$
v_{x_{\infty}}\left(d\left(\frac{1}{x^{a} y^{b}}\right)\right)=v_{x_{\infty}}\left(\frac{b x^{a} y^{b-1} d y+a x^{a-1} y^{b} d x}{x^{2 a} y^{2 b}}\right)=0 .
$$

Используя равенство $d\left(y^{p}-y\right)=d f(x)$, или эквивалентное равенство $d y=-f^{\prime}(x) d x$, получаем, что

$$
v_{x_{\infty}}\left(\frac{\left(b x f^{\prime}(x)+a y\right) d x}{x^{a+1} y^{b+1}}\right)=0 .
$$

Поскольку

$$
\begin{aligned}
v_{x_{\infty}}\left(b x f^{\prime}(x)+a y\right) & =\min \left\{v_{x_{\infty}}\left(b x f^{\prime}(x)\right), v_{x_{\infty}}(a y)\right\} \\
& =v_{x_{\infty}}\left(b x f^{\prime}(x)\right)=-m p
\end{aligned}
$$

находим, что степень канонического делителя равна

$$
v_{x_{\infty}}(d x)=m p-m-p-1 \text {. }
$$

Таким образом, $2 g(X)-2=m p-m-p-1$, что приводит к равенству

$$
g(X)=\frac{1}{2}(p-1)(m-1) \text {. }
$$

Так как $\phi$ - покрытие степени $p$ проективной прямой $P^{1}$, получаем, что

$$
2 g(X)-2=p\left(2 g\left(P^{1}\right)-2\right)+\operatorname{deg} R_{\phi},
$$

где $R_{\phi}$ - делитель ветвления покрытия $\phi$.

Единственной точкой ветвления покрытия $\phi$ является точка $x_{\infty}$, поэтому $R_{\phi}=$ $a_{x_{\infty}} x_{\infty}$. Используя (4) и выражение для рода, находим, что $a_{x_{\infty}}=(p-1)(m+1)$.

Пусть теперь $A$ - линейное подпространство пространства $F_{q}[x]$. Пусть $\left\{f_{1}, \ldots, f_{s}\right\}$ - канонический базис подпространства $A$ такой, что $\operatorname{deg} f_{i}(x)=m_{i}$ и $\left(m_{i}, p\right)=1$. Предположим, что $m_{i}$ упорядочены так, что $m_{1} \leqslant \ldots \leqslant m_{s}$. Пусть $X_{s}$ - кривая, задаваемая над $\bar{F}_{q}$ расслоенным произведением

$$
\begin{gathered}
y_{1}^{p}-y_{1}=f_{1}(x) \\
y_{2}^{p}-y_{2}=f_{2}(x) \\
\cdots \\
y_{s}^{p}-y_{s}=f_{s}(x) .
\end{gathered}
$$

Теорема 3. Род кривой $X_{s}$, задаваемой (5), равен

$$
g\left(X_{s}\right)=p^{s-1} \frac{(p-1)\left(m_{s}-1\right)}{2}+\ldots+p \frac{(p-1)\left(m_{2}-1\right)}{2}+\frac{(p-1)\left(m_{1}-1\right)}{2} .
$$

Доказательство. Для $1 \leqslant r \leqslant s$ пусть $X_{r}$ - кривая в $P^{s-r+1}$, задаваемая системой (5) с удаленными первыми $r-1$ уравнениями. Тогда для любого $r, 2 \leqslant r \leqslant s, X_{r-1}$ есть покрытие $X_{r}$ и $X_{s}$ есть покрытие проективной прямой $P^{1}$, причем степень каждого из покрытий равна $p$. Таким образом, мы имеем цепочку покрытий

$$
X_{1} \rightarrow X_{2} \rightarrow \ldots \rightarrow X_{s} \rightarrow P^{1}
$$


Доказательство формулы для рода проведем индукцией по $s$. Для $s=1$ это утверждение содержится в лемме 1 . Предположим что оно верно для $s-1$, тогда

$$
g\left(X_{2}\right)=p^{s-2} \frac{(p-1)\left(m_{s-1}-1\right)}{2}+\ldots+p \frac{(p-1)\left(m_{2}-1\right)}{2}+\frac{(p-1)\left(m_{1}-1\right)}{2} .
$$

Поскольку $X_{1}$ есть покрытие степени $p$ для $X_{2}$, справедливо равенство

$$
2 g\left(X_{1}\right)-2=p\left(2 g\left(X_{2}\right)-2\right)+\operatorname{deg} R_{\phi_{2}}
$$

где $R_{\phi_{2}}$ - делитель ветвления покрытия $\phi_{2}: X_{1} \rightarrow X_{2}$. Имея в виду, что единственной точкой ветвления покрытия $\phi_{2}$ является точка на бесконечности и гладкая проективная кривая $X_{2}$ вблизи точки на бесконечности ведет себя как проективная прямая $P^{1}$, с помощью рассуждений, использованных в доказательстве леммы 1 , находим, что

$$
\operatorname{deg} R_{\phi_{2}}=(p-1)\left(m_{1}+1\right) .
$$

Таким образом, из (6) следует, что

$$
g\left(X_{1}\right)=p g\left(X_{2}\right)+\frac{1}{2}(p-1)\left(m_{1}-1\right),
$$

и теорема доказана.

Подчеркнем, что упорядочение $m_{1} \leqslant \ldots \leqslant m_{s}$ степеней полиномов $f_{1}(x), \ldots, f_{s}(x)$ существенно. Действительно, если рассматривать каждое уравнение $y_{i}^{p}-y_{i}=f_{i}(x)$ в (5) отдельно как покрытие проективной прямой и $x_{i_{\infty}}$ как обратный образ бесконечной точки $[0: 1] \in P^{1}$, то $x_{i_{\infty}}$ будет соответствовать такой дискретной норме $v_{x_{i_{\infty}}}$, что $v_{x_{i_{\infty}}}(x)=-m_{i}$. Таким образом, сначала придется рассматривать покрытие с минимальной нормой $x$ и ясно, что это есть покрытие с максимальной степенью $m_{i}$, скажем $m_{s}$. Проводя ту же операцию с заменой проективной прямой на кривую $y_{s}^{p}-y_{s}=f_{s}(x)$ и принимая во внимание, что гладкая проективная кривая вблизи точки на бесконечности ведет себя как проективная прямая, мы убеждаемся в необходимости упорядочения.

Следует отметить также, что для доказательства теоремы 3 можно было бы использовать формулу для рода элементарньх абелевьх $p$-расширений, приведенную в [1], или теорему 2.2 из [3], однако преимущества приведенного здесь доказательства заключаются в его простоте и замкнутости в смысле геометрии кривой $X_{s}$.

Замечание 4. Важно заметить, что значение рода, указанное в теореме 3 ограничено сверху величиной $\left(p^{s}-1\right)\left(m_{s}-1\right) / 2$.

\section{3. Число $F_{q}$-рациональных точек}

Пусть $p$ - простое число и $F_{q}$ - конечное поле с $q=p^{\nu}$ элементами. Обозначим $\operatorname{Tr}_{\nu}: F_{q} \rightarrow F_{p}$ функцию след. Число $N$ решений уравнения $y^{p}-y=f(x) \in F_{q}(x)$ в $F_{q} \times F_{q}$ задается формулой $N=p\left|\left\{x \in F_{q}: \operatorname{Tr}_{\nu} f(x)=0\right\}\right|$. Используя этот хорошо известный факт, получаем следующее утверждение.

Лемма 2. Пусть $p$ - простое число, $q=p^{\nu}$, где $\nu$ - положстельное целое число, u $F_{q^{2}}$ - конечное поле с $q^{2}$ элементами. Тогда для любого $a \in F_{q^{2}}^{*}$ такого, что $a+a^{q}=0$, ияобого челого $j \geqslant 1$ число $F_{q^{2}}$-рачиональньх точех аффинной кривой $y^{p}-y=a x^{j(1+q)}$ равно $q^{2} p$. 
Доказательство. Ясно, что

$$
\begin{aligned}
\operatorname{Tr}_{2 \nu}\left(a x^{j(1+q)}\right) & =\sum_{k=0}^{2 \nu-1}\left(a x^{j(1+q)}\right)^{p^{k}}=\sum_{k=0}^{\nu-1} a^{p^{k}} x^{j p^{k}(1+q)}+\sum_{k=\nu}^{2 \nu-1} a^{p^{k}} x^{j p^{k}(1+q)} \\
& =\sum_{k=0}^{\nu-1} a^{p^{k}} x^{j p^{k}(1+q)}+\sum_{k=0}^{\nu-1} a^{p^{k+\nu}} x^{j p^{k+\nu}(1+q)} \\
& =\sum_{k=0}^{\nu-1} a^{p^{k}} x^{j p^{k}(1+q)}+\sum_{k=0}^{\nu-1}-a^{p^{k}} x^{j p^{k}\left(q+q^{2}\right)}
\end{aligned}
$$

Для любого $x \in F_{q^{2}}$ справедливо равенство $x^{q^{2}}=x$, поэтому $\operatorname{Tr}_{2 \nu}\left(a x^{j(1+q)}\right)=0$ для любого $x \in F_{q^{2}}$. Отсюда следует утверждение леммы.

Лемма 3. Пусть $F_{q^{2}}-$ то же, что $и$ в лемме 2. Тогда для ґюбого $a \in F_{q^{2}}^{*} u$ любого челого $j, 0<j \leqslant \nu-1$, число $F_{q^{2}}$-рачиональных точех аффинной кривой

$$
y^{p}-y=a x^{1+p^{\nu+j}}-a^{p^{\nu-j}} x^{1+p^{\nu-j}}
$$

равно $q^{2} p$.

Дохазательство. Ясно, что

$$
\begin{aligned}
\operatorname{Tr}_{2 \nu}\left(a x^{1+p^{\nu+j}}-a^{p^{\nu-j}} x^{1+p^{\nu-j}}\right) & \\
= & \sum_{k=0}^{2 \nu-1}\left(a x^{1+p^{\nu+j}}\right)^{p^{k}}-\sum_{k=0}^{2 \nu-1}\left(a^{p^{\nu-j}} x^{1+p^{\nu-j}}\right)^{p^{k}} \\
= & \sum_{k=0}^{\nu-j-1}\left(a x^{1+p^{\nu+j}}\right)^{p^{k}}+\sum_{k=\nu-j}^{2 \nu-1}\left(a x^{1+p^{\nu+j}}\right)^{p^{k}} \\
& \quad-\sum_{k=0}^{\nu+j-1}\left(a^{p^{\nu-j}} x^{1+p^{\nu-j}}\right)^{p^{k}}-\sum_{k=\nu+j}^{2 \nu-1}\left(a^{p^{\nu-j}} x^{1+p^{\nu-j}}\right)^{p^{k}} \\
= & \sum_{k=0}^{\nu-j-1} a^{p^{k}} x^{p^{k}\left(1+p^{\nu+j}\right)}+\sum_{k=0}^{\nu+j-1} a^{p^{\nu-j+k}} x^{p^{k}\left(p^{\nu-j}+q^{2}\right)} \\
& -\sum_{k=0}^{\nu+j-1} a^{p^{\nu-j+k}} x^{p^{k}\left(1+p^{\nu-j}\right)}-\sum_{k=0}^{\nu-j-1} a^{p^{2 \nu+k}} x^{p^{k}\left(p^{\nu+j}+q^{2}\right)} \\
= & \sum_{k=0}^{\nu-j-1} a^{p^{k}} x^{p^{k}\left(1+p^{\nu+j}\right)}-\sum_{k=0}^{\nu-j-1} a^{p^{2 \nu+k}} x^{p^{k}\left(p^{\nu+j}+q^{2}\right)} \\
& +\sum_{k=0}^{\nu+j-1} a^{p^{\nu-j+k}} x^{p^{k}\left(p^{\nu-j}+q^{2}\right)}-\sum_{k=0}^{\nu+j-1} a^{p^{\nu-j+k}} x^{p^{k}\left(1+p^{\nu-j}\right)} .
\end{aligned}
$$

Используя то, что $a^{q^{2}}=a$ и $x^{q^{2}}=x$ для всех $x \in F_{q^{2}}$, получаем, что

$$
\operatorname{Tr}_{2 \nu}\left(a x^{1+p^{\nu+j}}-a^{p^{\nu-j}} x^{1+p^{\nu-j}}\right)=0
$$

для любого $x \in F_{q^{2}}$. Отсюда следует утверждение леммы. 
Пусть $S_{1}, S_{2} \subset F_{q}[x]-$ множества полиномов, соответственно, вида

$$
f_{1}(x)=a_{i} x^{j(1+q)}
$$

где $a_{i} \in F_{q^{2}}$ удовлетворяют соотношению $a_{i}^{q}+a_{i}=0$ и $F_{p}$-линейно независимы и $1 \leqslant j<q,(j, p)=1 ;$ и

$$
f_{2}(x)=a_{i} x^{1+p^{\nu+j}}-a_{i}^{p^{\nu-j}} x^{1+p^{\nu-j}}
$$

где $a_{i}$ принадлежат $F_{q^{2}}$ и $F_{p}$-линейно независимы и $1 \leqslant j \leqslant \nu-1, i=1,2$.

Используя эти множества и леммы 2 и 3 , получаем следующий результат.

Лемма 4. Пусть $F_{q^{2}}$ - то же, ито $и$ в леммах 2, 3. Пусть $s, 1 \leqslant s<q$, челое число тахое, что $(s, p)=1, u X_{s}$ - аффинная кривал, определеннал над $\bar{F}_{q^{2}}$ раслоенным прочзведением

$$
\begin{aligned}
y_{1}^{p}-y_{1} & =f_{1}(x) \\
y_{2}^{p}-y_{2} & =f_{2}(x) \\
& \ldots \\
y_{s}^{p}-y_{s} & =f_{s}(x),
\end{aligned}
$$

где $f_{i}(x) \in S_{1} \cup S_{2}$ имент степени $\operatorname{deg} f_{i}(x) \leqslant s(q+1), i=1, \ldots, s$.

Тогда число $F_{q^{2}}$-рачионалъных точек $N_{q^{2}}\left(X_{s}\right)$ кривой $X_{s}$ равно

$$
N_{q^{2}}\left(X_{s}\right)=q^{N_{1}(s)+2}
$$

где $N_{1}(s)=s-[s / p]+2\left[\log _{p}((s(q+1)-1) / q)\right]$.

Дохазателъство. Семейство полиномов $S_{1} \cup S_{2}$ образует канонический базис невырожденного линейного подпространства пространства $F_{q}[x]$, так что данная система определяет кривую. В силу лемм 2 и 3 для любого $x \in F_{q^{2}}$ расслоение над $x$ на кривой $X_{s}$ содержит $p^{N(s)} F_{q^{2}}$-рациональных точек, где $N(s)$ - число определяющих уравнений этой кривой. Записывая $s$ в виде $s=n p+r, 1 \leqslant r \leqslant p-1$, видим, что число цельг $j$ таких, что $1 \leqslant j \leqslant s$ и $(j, p)=1$, равно

$$
n(p-1)+r=n p+r-n=s-n=s-[s / p] \text {. }
$$

Таким образом, число определяющих уравнений для полиномов из $S_{1}$ равно $\nu(s-[s / p])$. Число определяющих уравнений для полиномов из $S_{2}$ равно $2 \nu m$, где $m$ - наибольшее целое число такое, что

$$
p^{\nu+m}+1 \leqslant s\left(p^{\nu}+1\right) .
$$

Таким образом,

$$
m=\left[\log _{p}((s(q+1)-1) / q)\right]
$$

и, следовательно,

$$
N(s)=\nu\left((s-[s / p])+2\left[\log _{p}((s(q+1)-1) / q)\right]\right)=\nu N_{1}(s),
$$

что завершает доказательство. 
Две следующие леммы относятся к случаю $F_{q}, q=p^{2 \nu+1}$.

Лемма 5. Пусть $p$ - простое число, $q=p^{2 \nu+1}$, әде $\nu$ - положсительное целое число, и $F_{q}$ - конечное поле с $q$ элементами. Тогда для любого $a_{i} \in F_{q}^{*} u$ яюбого целого $j \geqslant 1$ число $F_{q}$-рачиональны

$$
y^{p}-y=a_{i} x^{j\left(p^{\nu+1}+1\right)}-a_{i}^{p^{\nu}} x^{j\left(p^{\nu}+1\right)}
$$

равно $q p$.

Доказательство. Ясно, что

$$
\begin{aligned}
& \operatorname{Tr}_{2 \nu+1}\left(a x^{j\left(p^{\nu+1}+1\right)}-a^{p^{\nu}} x^{j\left(p^{\nu}+1\right)}\right) \\
& =\sum_{k=0}^{2 \nu}\left(a x^{j\left(p^{\nu+1}+1\right)}\right)^{p^{k}}-\sum_{k=0}^{2 \nu}\left(a^{p^{\nu}} x^{j\left(p^{\nu}+1\right)}\right)^{p^{k}} \\
& =\sum_{k=0}^{\nu-1} a^{p^{k}} x^{j p^{k}\left(p^{\nu+1}+1\right)}+\sum_{k=\nu}^{2 \nu} a^{p^{k}} x^{j p^{k}\left(p^{\nu+1}+1\right)} \\
& -\sum_{k=0}^{\nu} a^{p^{\nu+k}} x^{j p^{k}\left(p^{\nu}+1\right)}-\sum_{k=\nu+1}^{2 \nu} a^{p^{\nu+k}} x^{j p^{k}\left(p^{\nu}+1\right)} \\
& =\sum_{k=0}^{\nu-1} a^{p^{k}} x^{j p^{k}\left(p^{\nu+1}+1\right)}+\sum_{k=0}^{\nu} a^{p^{\nu+k}} x^{j p^{\nu+k}\left(p^{\nu+1}+1\right)} \\
& -\sum_{k=0}^{\nu} a^{p^{\nu+k}} x^{j p^{k}\left(p^{\nu}+1\right)}-\sum_{k=0}^{\nu-1} a^{p^{2 \nu+k+1}} x^{j p^{\nu+k+1}\left(p^{\nu}+1\right)} \\
& =\sum_{k=0}^{\nu-1} a^{p^{k}} x^{j p^{k}\left(p^{\nu+1}+1\right)}+\sum_{k=0}^{\nu} a^{p^{\nu+k}} x^{j p^{k}\left(p^{2 \nu+1}+p^{\nu}\right)} \\
& -\sum_{k=0}^{\nu} a^{p^{\nu+k}} x^{j p^{k}\left(p^{\nu}+1\right)}-\sum_{k=0}^{\nu-1} a^{p^{2 \nu+k+1}} x^{j p^{k}\left(p^{2 \nu+1}+p^{\nu+1}\right)}
\end{aligned}
$$

Используя то, что $a^{q}=a$ и $x^{q}=x$ для всех $x \in F_{q}$, получаем, что

$$
\operatorname{Tr}_{2 \nu+1}\left(a x^{j\left(p^{\nu+1}+1\right)}-a^{p^{\nu}} x^{j\left(p^{\nu}+1\right)}\right)=0
$$

для всех $x \in F_{q}$, что завершает доказательство.

Взяв $a_{i}$ в предшествующей лемме так, что они образуют базис $F_{q}$ над $F_{p}$ и рассуждая так, как в доказательстве леммы 4, получаем следующее утверждение.

Лемма 6. Пусть $F_{q}$ - то же, что и в лемме 5. Тогда для любого челого $s$, $1 \leqslant s<p^{\nu-1},(s, p)=1$, расслоенное произведение

$$
y_{i j}^{p}-y_{i j}=a_{i} x^{j\left(p^{\nu+1}+1\right)}-a_{i}^{p^{\nu}} x^{j\left(p^{\nu}+1\right)}
$$

где $1 \leqslant j \leqslant s,(j, p)=1$, uмеem

$$
q p^{(2 \nu+1)(s-[s / p])}
$$

$F_{q}$-рачиональных точек. 


\section{4. Доказательство теорем}

В этом параграфе доказывается теорема 1 и следствие 1. Доказательства теоремы 2 и следствия 2 совершенно аналогичны.

Доказательство теоремъ 1. Пусть $F_{q^{2}}$ - конечное поле с $q^{2}=p^{2 \nu}$ элементами и $1 \leqslant s<q,(s, p)=1$. Пусть $X_{s}$ - аффинная кривая, определенная в лемме 4 . Согласно этой лемме число $F_{q^{2}}$-рациональных точек в $X_{s}$ равно $q^{N_{1}(s)+2}$, где

$$
N_{1}(s)=s-[s / p]+2\left[\log _{p}((s(q+1)-1) / q)\right] .
$$

Нормализуя кривую $X_{s}$, получаем несингулярную модель $\tilde{X}_{s}$ без нарушения $F_{q^{2}-}$ рациональности этих точек. Принимая во внимание точку на бесконечности $p_{\infty}$ кривой $\tilde{X}_{s}$, находим, что

$$
N_{q^{2}}\left(\tilde{X}_{s}\right)=q^{N_{1}(s)+2}+1 .
$$

Согласно теореме 3 и следствию 4 род кривой $\tilde{X}_{s}$ не превосходит

$$
\frac{1}{2}\left(q^{N_{1}(s)}-1\right)(s(q+1)-1),
$$

что и завершает доказательство.

Прежде чем приступить к доказательству следствия 1 , напомним известную конструкцию линейных $[n, k, d]_{q}$-кодов Гоппы, связанньх с гладкой проективной кривой $X$ над конечным полем $F_{q}$, где $q=p^{\nu}$ (см. [2]).

Пусть $X$ - абсолютно неприводимая гладкая проективная кривая рода $g$ над $F_{q}$. Пусть $\left\{p_{1}, \ldots, p_{n}\right\}-$ множество различных $F_{q}$-рациональных точек на $X$ и $D_{0}=$ $p_{1}+\ldots+p_{n}$. Пусть $D-F_{q}$-рациональный делитель на $X$ такой, что носители $D$ и $D_{0}$ не пересекаются. Линейное пространство

$$
L(D)=\left\{f \in F_{q}(X)^{*} \mid(f)+D \geqslant 0\right\} \cup\{0\}
$$

порождает линейное отображение

$$
E v: L(D) \rightarrow F_{q}^{n}, \quad f \rightarrow\left(f\left(p_{1}\right), \ldots, f\left(p_{n}\right)\right) .
$$

Образ этого отображения есть линейный $[n, k, d]_{q}$-код $C=C\left(D_{0}, D\right)$, называемый геометрическим кодом Гоппы, связанным с парой $\left(D_{0}, D\right)$.

Если $\operatorname{deg} D<n$, то $E v$ есть вложение, поэтому $k=\operatorname{dim} C=l(D)$. По теореме Римана-Poха

$$
k=l(D) \geqslant \operatorname{deg} D-g+1 \text {. }
$$

В частности, если $2 g-2<\operatorname{deg} D<n$, то $k=\operatorname{deg} D-g+1$.

Минимальное расстояние $d$ кода $C=C\left(D, D_{0}\right)$ удовлетворяет неравенству

$$
d \geqslant n-\operatorname{deg} D \text {. }
$$

Отсюда следует, что относительные параметры $R=k / n$ и $\delta=d / n$ линейного $[n, k, d]_{q}$-кода $C=C\left(D_{0}, D\right)$ удовлетворяют соотношению

$$
R \geqslant 1-\delta-\frac{g-1}{n}
$$

Теперь, применяя конструкцию Гоппы к кривым из теоремы 1 , мы можем доказать следствие 1. 
Доказательство следствия 1. Пусть $X_{s}$ - гладкая проективная кривая, определенная в теореме 1 . Пусть $S$ - множество $F_{q^{2}}$-рациональных точек на конечной части кривой $X_{s}$ и $p_{\infty}$ - точка кривой $X_{s}$ на бесконечности. Согласно лемме 4 $|S|=q^{N_{1}(s)+2}$. Пусть $n \leqslant q^{N_{1}(s)+2}$ и

$$
D_{0}=p_{1}+\ldots+p_{n}, \quad D=l p_{\infty} .
$$

Применяя конструкцию Гоппы к этим $F_{q^{2}}$-делителям на $X_{s}$ для каждого $l$ такого, что

$$
\frac{1}{2}\left(q^{N_{1}(s)}-1\right)(s(q+1)-1)<l<n,
$$

мы получаем требуемый код.

\section{Список литературы}

1. Garcia A., Stichtenoth H., Elementary abelian p-extensions of algebraic function fields. Manuscr. Math. (1991) 72, 67-79.

2. Goppa V. G., Codes on algebraic curves. Soviet Math. Dokl. (1981) 24, 170-172.

3. Lachaud G., Artin-Schreier curves, exponential sums, and the Carlitz-Uchiyama bound for geometric codes. J. Number Theory (1991) 39, 18-40.

4. Özbudak F., Codes on fibre products of some Kummer coverings. Finite Fields and their Appl. (1999) 5, 188-205.

5. Степанов С. А., Арифметиха алгебрачиеских кривых. Наука, Москва, 1971.

6. Stepanov S. A., Codes on Algebraic Curves. Plenum, New York, 1999.

7. Степанов С. А., Коды на расслоенных произведениях гиперэллиптических кривых. Дискретная математика (1997) 9, №1, 83-94.

8. Степанов С. А., Озбудак Ф., Расслоенные произведения гипергеометрических кривых и геометрические коды Гоппы. Дискретная математиха (1997) 9, №3, 36-42.

9. G. van der Geer, M. van der Vlugt, How to construct curves over finite fields with many points. Algebraic Geometry E-prints, alg-geom/9511005, 1995.

10. Xing C., Stichtenoth H., The genus of maximal function fields. Manuscr. Math. (1955) 86, 217-224. 